\title{
An Comprehensive Survey on Applications of Precision Agriculture in the Context of Weed Classification, Leave Disease Detection, Yield Prediction and UAV Image Analysis
}

\author{
Preethi $\mathrm{C}^{\mathrm{a}, 1}$, Brintha $\mathrm{NC}^{\mathrm{b}}$ and Yogesh $\mathrm{CK}^{\mathrm{c}}$ \\ ${ }^{a}$ Research Scholar, Department of Computer Science and Engineering, Kalasalingam \\ Academy of Research and Education, Krishnankoil, India \\ ${ }^{\mathrm{b}}$ Associate Professor, Department of Computer Science and Engineering, Kalasalingam \\ Academy of Research and Education,Krishnankoil, India \\ ${ }^{\mathrm{c}}$ Assistant Professor,Department of Computer Science and Engineering, Vel Tech \\ Rangarajan Dr.Sagunthala R\& D Institute of Science and Technology, Chennai, India
}

\begin{abstract}
Advancement in technologies such as Machine vision, Machine Learning, Deep Learning algorithms enables them to extend its horizon in different applications including precision agriculture. The objective of this work is to study the various works pertaining to precision agriculture under four categories, weed classification, disease detection in leaves, yield prediction and image analysis techniques in UAV. In case of the weed classification, both classifying weeds from the crops and classifying the different types of weeds are analysed. In disease detection, only the diseases that occur in the leaves of different plants are considered and studied. It is continued with the state of art models that predicts yields of different crops. The last part of the work concentrates on analysing the images captured UAV in the context of precision agriculture. This work would pave a way for getting a deep insight about the state of art models related to the above specified applications of precision agriculture and the methods of analysing the UAV images.
\end{abstract}

Keywords. precision agriculture, weed classification, yield prediction, disease detection.

\section{Introduction}

The following can be inferred from the definition of the precision culture provided by international society of precision agriculture. Different data are collected from different sources through different tools; this data includes spatial data, temporal data, and individual data. Decisions were made on the basis of the data gathered and other available

\footnotetext{
${ }^{1}$ Preethi C, Department of CSE, Kalasalingam Academy of Research and Education, Krishnankoil, India. E-mail: preethic20@gmail.com.
} 
information to improve the overall process in terms of various parameters. According to the objectives of the precision agriculture is to Improving the product's quality, Increase the Productivity, Energy optimization, Optimized use of products like fertilizers and pesticides and to take measures against pollution. The concept of precision agriculture dated back to 1980's is enabled with different techniques such as Global Positioning System, Geographic information system, remote sensing, Wireless sensor networks, unmanned aerial vehicles and Machine vision [1]. Though there are various applications based on precision agriculture, this work concentrates on making a deep survey on precision agriculture in the following perspectives such as Weed Classification, Leaf disease detection, Yield prediction and Image analysis in UAV. The papers that were analysed include the models that were used in various form of precision agriculture. A separate section for studying the state of art models that analysis images captured by UAV is also given in addition to the various models.

\section{Weed classification}

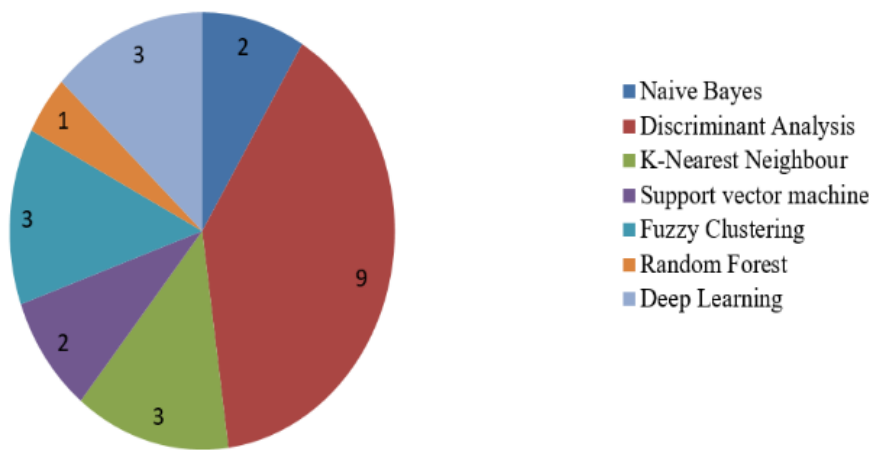

Figure 1. Summary of models for weed Detection

Weeds are wild plants that grow in between the cultivated plants; the process of detecting the weeds is an important process in agriculture. There are various weed detection mechanisms that employs various machine learning and deep learning models. They are Naïve Baiyes, Discriminant Analysis,K-Nearest Neighbour, Support Vector Machine, Fuzzy Clustering, Random Forest and Deep Learning. The weed classification can be divided into two such as Classification of weeds and crops and Classification of weeds.

\subsection{Classification of weeds and crops}

(A. Piron et al. 2009) states that in the earlier studies the height of the crops and the multispectral features are considered individually for classification of plants and the weeds with the help of quadratic discriminant analysis. The authors have observed that employing both the features results only in slight increase in the classification accuracy. Hence the authors introduce a model for improving the classification accuracy by introducing 
a threshold between the height of the weeds and the height of the crops. With the introduction of the model, the classification accuracy achieved is $82 \%$.

Segmentation of the plant species from the soil and classification of plants and different kinds of weeds are performed in (Okamoto, $\mathrm{H}$ et al. 2007)through Euclidean distance and the discriminant analysis. The later model uses wavelet coefficients .step by step discriminant analysis performs well in classifying the plants and the weeds than the model that employs Euclidean distance. (Kazmi, W et al. 2015) have proposed a model for classifying the thistles from the sugar beet fields. Around 14 features were employed by the authors and two models called linear discriminant analysis and mahalanobis distance were employed. Different combinations of the features were employed and the best performing features are color index for vegetation extraction, excess green and green minus blue. This feature produces accuracies that were higher than $90 \%$. In another experiment the combination of the features excess green and green minus blue produces a better accuracy of $95 \%$.

Identification of weeds and classification of tomato leaves were done in (Lee, W. S. et al. 2004) Initially the images are classified in to regions with plants and without plants. The features that were considered for this classification are saturation, hue and intensity. Bayesian decision model is used for this classification. This process is continued by the identification of leaves which is done through the series of steps that result in removal of noise. Noise removal is made with processes called as shrinking followed by swelling. Plant recognition procedure is applied and the leaves are classified to either good or bad initially. For this purpose, around 10 shape features are considered. After the leaves are classified into good or bad, it is classified in to four classifications, cotyledon, tomato leaves- true/miscellaneous and weeds. Here also Bayesian discrimination is used. The accuracy obtained in identification of the weeds is only $48 \%$.

(Muhammad Hamza Asad and Abdul Bais 2019) have proposed a model for classifying weeds in the canola fields. The process of segmenting the plants and the vegetation is done with maximum likelihood model. After this segmentation, manual labelling of pixels indicating weeds is done for further training and classification. Different deep learning models are employed and the performances are evaluated. UNET_VGG16, SegNet_VGG16, UNET_ResNet-50,SegNet_ResNet-50 are used, though the overall accuracies are higher the performance in detecting the weed is low.The performance metric used for measuring the identification of weed is intersection over union.

Carrots and weeds are classified in (Florian J. Knoll et al. 2019) with deep convolutional neural networks, though decreasing the computation time is the objective of the work, the overall classification accuracy obtained by the authors is $99 \%$. The accuracy of identifying the weed detection is $93.12 \%$ in conventional model and the accuracy value decreases in the optimized model to $86.88 \%$.

(Astrand.B and Baerveldt.A 2002) designed a mechanical robot that is used for detecting the weeds among the sugar beets. Histogram thresholding is used for segmenting the plants from the rest of the image. This is followed by extracting the features; the features include colour, shape and moment-based features. KNN is used for classifying the weeds from the sugar beets. The accuracy obtained is 97\%. (Ahmed.F et al. 2012) have proposed a model for classifying weeds from the chilli plants using support vector machine. The image processing steps includes image segmentation by means of binarization, it is followed by morphological opening for removing the noise. The features 
that were extracted are moment invariants, shape features that are independent of the size and the colour features. $97 \%$ accuracy is obtained with SVM.

Random forest classifier and soft independent modelling by class analogy is employed in (Micael Felipe de Souza et al. 2020) for classifying weeds from the sugar cane. Random forest model performs better than the other models and produces an accuracy of 99.5\% in classifying the weeds from the crops. (Tellaeche, A et al. 2007) have produced a model to classify weeds from the plants, in this approach, binarized images are created from the RGB images using thresholding approach. Morphological opening is used for removing unwanted pixels. From the resulting image, extraction of Area based attributes is done and it is given as input for the Bayesian and fuzzy K-means for classification. The accuracy obtained is $91 \%$.

\subsection{Classification of weeds}

Meyer, $\mathrm{G}$ et al. 1998 have proposed a model for differentiating the grass/weeds from the soil . The experimentation is done with two kinds of leaves and two kinds of weeds. In order to segment the soil and the plant, as a first step, sub images were generated by the authors each one for red, green and the blue. From the colour model, the values are normalized and average excess green image is computed. It has been observed that the pixels representing the soil are not as brighter as the pixels that represent the plants. In order to make the discrimination between the plants and the soils and the discrimination between the plants, texture features are obtained from pixels of plant and soil. The features that were considered are excess green, local homogeneity, entropy, inertia and angular second moment. The result obtained is high (99\%) for soil discrimination and it is low for classifying the species (30\% to $70 \%)$. Also the author from

Classification of six types of weeds is done in Burks, T et al which employed by the authors is colour co-occurrence method. The RGB images are converted to HSI images from which the CCM matrices are generated. The texture properties are extracted from it and CCM statistical methods were applied for classification. The accuracy obtained with weed classification is $93 \%$.

Weed classification has been done in (Ahmad. I et al 2011) with K-nearest neighbour classifier enabled with Haar Wavelet transform. The work includes three processes segmentation by which the unwanted regions are removed; extraction of features with Haar wavelet transform by means of which 200 features were extracted and finally classification is done. The features extracted are texture features and it includes horizontal, diagonal and vertical coefficients. Classification is done with KNN classifier and the accuracy obtained is $94 \%$.

A mechanism has been proposed in (Pereira, L.A et al. 2012) for identifying aquatic weeds. Image segmentation is applied for extracting the similar regions from the images. The exact shape of the leaves is extracted by further processing called filling regions and elimination of connected components. The image segmentation is followed by shape analysis and classification. Five feature extraction models are employed and different classifiers such as optimum path classifier, support vector machine, Bayesian classifier, multilayer perception and self organizing map are employed. The best accuracy is achieved with optimum path classifier with beam angle statistics extractor.

Apart from both these kinds of classifications there are certain works that concentrates on finding the weed density, classifying the weeds from grasses in grass lands. Such works are described below. 
A model has been proposed in (Gebhardt Steffen et al. 2006) for identifying a particular kind of weeds in grasslands. The leaves of the plants and the leaves of the weeds are distinguished from the background by the authors through homogeneity measurement. With the homogeneity threshold binary images were formed by the authors. The features that were considered for classification are colour, shape and texture. Maximumlikelihood is employed for classifying the weeds and the accuracy obtained varies from 71 to $95 \%$. Weed density is measured in (Solahudin, Mohamad et al. 2010) with the help of RGB colour components. The weed density is defined by the features intensity, saturation and hue. The weeds and the plants are categorized based on the fractal dimensions. The authors have presented the results in terms of the values of the fractal dimension of the plants and the weeds.

(Neto, J.C et al. 2006) have used the identification of individual leaves as a mean of classifying the weeds and the other plants. Technologies, Gustafson-Kessel clustering, genetic algorithm are used for extraction of the leaves. The outcome of the research is given in terms of the percentage of the leaf extraction.

Two datasets were considered in (Alessandro dos Santos Ferreira et al. 2019) one that is captured in soybean field and the dataset is used for discriminating between the grass and the broad leafs. The second dataset contains eight types of weeds and it is used for discriminating between the weeds. Two models are employed, first one uses Deep Clustering for making clusters of Visual Features and it is called as DeepCluster and the second model is called as JULE which stands for Joint Unsupervised Learning of Deep Representations and Image Clusters . Both of these clustering models are based on Alexnet and VGG-16 convolutional neural network. It is also observed that incorporation of transfer learning increases the accuracy of the model.

\section{Disease detection}

Disease detection is the process of identifying the diseases that occurs in various parts of the leaves [2]. Studies have shown that various classification algorithms are in use for disease classification. Disease detection can be made in Leaves, Fruits and Other parts of the plants The one that is concentrated in this study is the leaves disease detection. The study includes both the machine learning and deep learning models. Fluorescence features extracted by fluorescence sensors are used for identifying the Huanglongbing disease in citrus leafs in Sankaran. S and Ehsani. The machine learning models employed are naive bayes classifier and bagged decision tree classifier. While the naive bayes classifier performs well in the laboratory condition,bagged decision tree outperforms it in the field. The overall accuracy obtained with the later model is $94 \%$.

Features like hue, intensity and saturation are extracted and analysed with color co-occurence model. this is used for classifying the citrus leaves as scab, greasy spot, melanose and normal in (Bandi et al 2013). Four classifier models are employed Random Forest, KNN, Naive Bayes and linear discriminate analysis. Linear discriminate analysis performs well than the other models with an accuracy of 98.75

Yellow vein mosaic virus disease is identified in (Mondal, D et al. 2017). Morphological features are extracted from okra leaves and bitter gourd leafs. From the okra leaves ten key features are extracted and from bitter gourd leaves 9 key features are extracted by the authors. 95\% accuracy is obtained in case of identifying yellow vein mosaic virus disease in okra and the accuracy obtained in case of bitter gourd is $82.67 \%$. 
Continuous wavelet analysis is used in (Zhang, $J$ et al. 2017) for identifying and discriminating diseases and the damages that are found in the leaves. Fisher's linear discriminant analysis (FLDA) is employed for classifying the disease. This is based on the wavelet scalogram that are continuously decomposed the features that are employed are wavelet features. The overall accuracy obtained is $77 \%$.

Disease classification of three types of disease are made in the leaves and canopies in (Shi, Y et al. 2017). The model used by the authors is kernel discriminant analysis. Spectral features were used for discrimination. The accuracies obtained are given in leaf level and canopy level. In the former case the accuracy ranges from $76 \%$ to $95 \%$ and in the later case, the accuracy is greater than $87 \%$.

Multi band optical sensor is used in (Mishra, A et al. 2011) for identifying the Huanglongbing disease in the citrus leaves. Reflectance of the canopy is measured in four different bands and five classification algorithms are employed, decision trees, KNN, Logistic Regression, Neural Networks and support vector machines. Results depicts that SVM has lower classification error.

989 spectral features were collected in (Sankaran, S et al. 2011) from the spectral reflectance data with visible-near infrared spectroscopy. The number of features is reduced to 86 with the help of PCA. quadratic discriminant analysis (QDA), linear discriminant analysis, soft independent modelling of classification analogies(SIMCA) and $\mathrm{k}$-nearest neighbour are the algorithms that are employed for classification.Comparative result analysis shows that quadratic discriminant analysis provides better result.

Two types of diseases canker and Huanglongbing are identified in (Sankaran, S et al. 2013) with the help of the spectral features. Both mid infrared spectroscopy and visiblenear infrared spectroscopy are employed for getting the spectral images. Two types of classifiers are employed, K-Nearest neighbour and Quadratic discriminant analysis. Both produces accuracy greater than $90 \%$.

Two kinds of apple leaf diseases called as alternaria and miner pest were detected in (Omrani, E et al. 2014) with classification algorithms, support vector regression machine and artificial neural networks. The infected regions of the plant leaves are first detected with K-means clustering followed by extraction of colour, texture and shape features. Then the classifiers are applied. Support vector regression model performs well than the artificial neural network model. Two kinds of kernels are experimented in support vector regression model, RBF and polynomial. The performance parameter considered for evaluating the models is coefficient ofdetermination. It is inferred that support vector regressor with RBF kernel performs well than the other models. Co-occurrence and texture features are obtained from the leaf images. The extracted features are fed to two classifiers as input, the classifiers are SVM and minimum distance criterion (Arivazhagan, S et al. 2013). The classification accuracy obtained in the formal model is $86.77 \%$ and the accuracy obtained in the later model is $94.74 \%$.

Classification of diseases such as Southern green stink bug, Bacterial angular, Ascochyta blight are classified in (Camargo, A et al. 2009) with the help of the following features. shape, texture, lacunarity, dispersion, fractal dimension, grey levels, grey histogram discrimination and fourier descriptor. These features are given as input to the support vector machine for classification. Experimentation is done with different combination of features and the best result is achieved with all features.

Diseases such as the scab disease and the rust disease that occurs in grape leaves are identified in (Meunkaewjinda, A et al. 2008). The pre processing work includes the 
segmentation of the grape leaves, the affected regions of the leaves are segmented with the help of the self organizing map and genetic algorithm . The same is used for optimization. Finally support vector machine is used for grape leaves classification.

SVM is used in (Sannakki, S.S et al. 2013) for classifying the disease that occurs in pomegranate leaves. K-means clustering is used for segmenting the colour images and the result is fed to SVM. The features extracted and given as input to the classifier are hue, energy, saturation and entropy. The accuracy obtained is $97.30 \%$.

95\% accuracy is achieved in (Islam, $\mathrm{M}$ et al. 2017) for classifying the potato leaves that has disease and that does not have. Plant village dataset is used for evaluating the model. The leaf region is extracted and the colour and the texture features are got from it. The features are given as input to the support vector machine. The disease classified is blight disease either in early or later stage. The spots representing the disease in the rice plant leaves are identified by extracting the colour features, texture features and shape features. The features were given as inputs to the support vector machine. The overall accuracy obtained in (Yao, Q et al. 2009) is 97.2Colour co-occurrence method is used for extracting the texture features from the leaves in (Singh, V and Misra, A.K 2017) and given as input to the support vector machine. Support vector machine classifier is built to identify various types of disease that occurs in leaves, such as scorch disease, sun burn, bacterial leaf spot and fungal diseases. Experimentation has been done with leaves from various plants. The average accuracy obtained is 97.6Classification of normal tomato leaves and healthy tomato leaves is made in (Mokhtar, $U$ et al. 2014). Nine texture features are extracted and gray level co-occurence matrix is used for generating 36 texture features. The features are given as input to SVM and the highest accuracy obtained is 99.83In (Xiaoling Deng et al. 2019) Primary spectral data is considered as the input for the classification algorithms designed for classifying the Huanglongbing disease that occurs in the citrus fruit. Entropy distance band selection method and sequential backward selection is used for optimization the results of which are compared with the results obtained with principal component analysis. Three different datasets were created with different features and 6 different machine learning algorithms are applied for classification. The maximum accuracy is achieved in support vector machine with principal component analysis for feature selection. Citrus greening disease also known as huanglongbing is a commonly occurring disease in citrus plants. The preprocessing steps include removal of noise, calculation of vegetation index, incorporation of principal component analysis for feature compression and incorporating autoencoders for identifying the features. SVM, kNN, logistic regression (LR), naive Bayes and ensemble learning models are used .100\% accuracy is achieved with adaboost in (Yubin Lan et al. 2020). In case of (Wan-Soo Kim et al. 2020) weakly supervised learning model is used for object localization as a pre processing step of identifying the onion downy disease. Class activation map is used by the authors for visualizing the classified objects. $\mathrm{CNN}$ is employed for identifying the symptom of disease. The available VGG16 model is used to create 5 different models that are classified by the number of weight layers. The highest mean average precision obtained is 87.2.

Though the survey does not provide a complete list of all the works it can be inferred that support vector machine is used in most of the works for leaf disease detection and provides better results. The following table 1 summarizes it. Future researchers and works can consider support vector machine for their work in disease detection. 
Table 1. summarization of usage of support vector machine

\begin{tabular}{|l|l|}
\hline \multicolumn{2}{|l|}{ Support Vector Machine } \\
\hline Leaves & Diseases \\
\hline Citrus leaves & canker, Huanglongbing \\
\hline Apple leaves & alternaria, miner pest \\
\hline $\begin{array}{l}\text { Banana leaves, Rose leaves, Lemon } \\
\text { leaves, Beans leaves }\end{array}$ & $\begin{array}{l}\text { early scorch, yellow spots, brown spots, late scorch, bacterial } \\
\text { Diseases, fungal diseases }\end{array}$ \\
\hline Cotton leaves & Southern green stink bug, Bacterial angular, Ascochyta blight \\
\hline Grapes leaves & Scab, Rust \\
\hline Potato leaves & Blight \\
\hline Rice Plant & $\begin{array}{l}\text { Rice bacterial leaf blight (RBLB), rice sheath blight (RSB), } \\
\text { rice blast(RB) }\end{array}$ \\
\hline Tomato and pomegranate leaves & infected and damaged region \\
\hline
\end{tabular}

\section{Yield Prediction}

Another application of precision agriculture is yield prediction. The following is the summary of the few works that concentrates on yield prediction [3-5]. Most of the works are based on the images taken from UAV. In (Linghua Meng et al. 2019) date information of each pixel of the image is integrated with the NVDI values. Two different time series products are obtained with the aid of flexible spatiotemporal data fusion. The author specifies that with this the spatial effectiveness and the temporal effectiveness of the images obtained from the satellite are improved. Linear regression model is applied for different reconstructed data. The coefficient of determination value differs from 0.77 to 0.84. Eight different features extracted from the images taken with three different types of camera mounted in the UAV are used for estimating the cotton yield in (Aijing Feng et al. 2020). The eight factors are experimented separately and in different combinations. The best value of the coefficient of determination with individual parameter is 0.90 and it is achieved with both plant height and the cotton fibre index. The best coefficient of determination with two feature models ranges in the values from 0.92 to 0.94 .

In case of (Anna Mateo-Sanchis et al.2019), enhanced vegetation index and vegetation optical index are considered and combined to estimate the yield prediction of various crops. Two statistical models namely regularised linear regression and kernel ridge regression are used by the authors the results obtained varies depending on the crop and are considerably low. Maize yield prediction is made with the excess green colour feature in (Meina Zhang et al. 2020). Linear regression model is used for predicting the maize yield and the coefficient of determination obtained are less than 0.5. Six different features belonging to the vegetation indices and drought related indices are used as features for maize yield estimation in (Louise Leroux et al. 2019). With this features two models, multiple linear regression and random forest are employed for yield prediction. The performance of the Random forest model overcomes the performance of the multiple linear regression.

(Bo Li et al. 2020) used narrow band vegetation index as inputs for two different prediction models partial least square and random forest for predicting the potato yield. Rrelief feature selection algorithm is used for selecting two features among the different features. The prediction were made in two different time periods, one after 60 days of planting and another after 90 of planting . The performance parameters considered are 
coefficient of determination and RMSE . In case of partial least square the coefficient of determination obtained are 0.69 and 0.81 . The RMSE value is 0.56 and 0.42 . in case of random forest the values obtained for coefficient of determination is 0.44 and 0.63 and RMS is 0.73 and 0.63 .

Rice yield estimation is done in (Md Nasim Reza et al. 2019) by combining Kmeans clustering and a graph cut algorithm. These two algorithms are combined to segment the area of rice grain. K-means clustering is obtained and form the clustered image rice grain area is extracted with the help of the shoelace algorithm. The coefficient of determination obtained is 0.98. In (Petteri Nevavuori et al. 2019) RGB data and NDVI data obtained from the UAV is used as inputs to the convolutional neural network for crop prediction. The model is optimized in various ways such as use of Adadelta training algorithm and employment of 12 regularisation .the mean absolute error of this model is $484.3 \mathrm{~kg} / \mathrm{ha}$. The authors of (Maitiniyazi Maimaitijiang et al. 2020) Various features such as texture features, thermal features canopy spectral and structure are extracted from the images captured with UAV. various models such as random forest regression, Partial Least Squares Regression (PLSR), Support Vector Regression (SVR), input-level feature fusion and intermediate-level feature fusion based deep neural network models are employed for yield estimation. The last model performs well producing a coefficient of determination 0.720. Estimation of wheat ear is the task of (Simon Madec et al. 2019) and it is made with the help of R-CNN model the capability of the deep learning model to learn the important features by itself is used by the authors in this model and the root mean square error in this prediction is approximately 6

\section{UAV Image Analysis}

This section describes the cameras used in the drone, their applications and description of how images acquired from the drones $[7,8]$ are processed.Each camera has its own specific purpose as specified in (Pasquale Daponte et.al 2019) and is summarised in the Table 2 The objective of this section is to study and summarize the cameras used in the

Table 2. Applications of different cameras in UAV

\begin{tabular}{|l|l|}
\hline Camera & Application \\
\hline Multispectral camera & $\begin{array}{l}\text { Chlorophyll content, Leaf water content, Leaf area index, Nor- } \\
\text { malized difference vegetation index }\end{array}$ \\
\hline Thermal Camera & Water stress \\
\hline RGB cameras and LiDAR systems & Digitizing the terrain surface \\
\hline
\end{tabular}

drones and the pre-processing steps made with it . In (Aijing Feng et al. 2020) Three cameras are used in the model. a RGB camera, a multispectral camera and an infrared camera. Geo referenced images acquired from them are used to generate digital surface model with the aid of a method called as structure from motion method. Mosaic blending model is used for generating the orthomosaic images. Following this, fine tuning is performed row by row and then different features are extracted for yield estimation. In (Meina Zhang et al. 2020) were maize yield prediction is made, RGB images were collected from the with a camera during three different growth stages, kernel development, grain filling and physiological maturity.the Gegraphical positioning system of the UAV 
is used for geo-referencing the sequential images. Stitched panoramic images are created in the cloud server called as drone deploy. Since this work also concentrates on the impact of variable rate nitrogen application, geo-registration of $\mathrm{N}$ application data and geo-referenced yield data is also made. Various colour features are extracted and given as input to the regression model for yield estimation.

Two kinds of images RGB images and Hyperspectral images are used in (Bo Li et al. 2020) are used for finding the biomass and yield estimation. From the RGB images, RGB digital surface model and digital elevation model is acquired. From the hyperspectral images, vegetation indices are derived. With this features, random forest model is used for yield estimation and biomass estimation. In (Md Nasim Reza et al. 2019) RGB images were collected from UAV .from the captured images, mosaicked image is created, for further processing, the mosaicked image is cropped into cell images, each cell image is divided into sub-cell image. For individual sub cell image k-means clustering along with graph cut algorithm is used for getting the segmented image which has the rice grain image from which the yield would be calculated. Parrot's NIR-capable SEQUIOA-sensor is used in (Petteri Nevavuori et al. 2019) for acquiring spectral images. Images were captured in 9 different fields. These images are combined with the yield data and provided as input to the convolutional neural network. The training set and the test set are created with the processing such as mosaicing, sub sampling and shuffling. Multisensory, thermal and RGB sensors are used in the UAV employed in (Maitiniyazi Maimaitijiang et al. 2020) and the corresponding images are captured in the soybean field. Image acquisition is followed by Orthorectification and mosaicing. The other pre-processing steps includes, multispectral images are converted to reflectance, thermal image calibration. Various features are extracted from this for yield prediction [6].

In (Yubin Lan et al. 2020) green multispectral image, red multispectral images and as well as near infrared multispectral images of a citrus field is acquired from the UAV. These images are later used for disease detection. the pre-processing steps include removal of noise by means of linear stretching and region of interest extraction. Vegetation indices are then calculated to various machine learning algorithms for detecting citrus greening disease. Disease detection in soybean leaves are identified in (Willian Paraguassu Amorim et al. 2019) with the help of the digital negative images that are captured from drone. Simple linear iterative clustering is used for segmenting each plantation. 3000 superpixel images are generated from this which contains images pertaining to different classes. These are given as input to the convolutional neural network for disease detection.

Multispectral camera is used in the UAV for the process of counting the number of tress in (Lucas Prado Osco et.al. 2020) The pre-processing steps include orthorectification with reference to 9 ground control points. Radiometric corrections were made on the images with reference to the radiance values of the reflectance plate. The result of these steps is the orthorectified surface reflectance image from which tree locations can be identified. Citrus rootstock evaluation is made in (Yiannis Ampatzidis et al. 2019) with the help of the images captured with multispectral camera with five bands red, green, blue, red edge and near infrared. The process includes radiometric calibration. Aerial maps of each band are stitched together and given as input to the convolutional neural network for further processing. Himanshu Agrawal et al. (2019) stated that there are many sensors in precision agriculture which continuously monitor the soil, wind speed, camera and drone in the field. In (Muhammad Adeel Hassan et.al 2019) monitoring of NDVI is 
made during the different growth stages of the wheat. With this grain yield prediction is made. Sequoia 4.0 multi-spectral sensor is used for taking the spectral images. It consists of four monochrome sensors. The pre-processing steps includes Geo-referencing, dense point cloud building generating digital surface model and orthomosaic images. From this various vegetation indexes generated and yield prediction is made.

\section{Conclusion}

From the study the following observations were made, Segmenting plants from the background soil or any other kind of background is the foremost step in most of the precision agriculture applications. though there are various methods that employs various features for this segmentation process it is observed that simple techniques that incorporate Euclidean distance produces almost $100 \%$ accuracy.In case of classifying weeds from the crops commonly used features such as colour, shape, texture and moment invariants results in high accuracy greater than $97 \%$.Around $39 \%$ of the works studied for weed classification belongs to discriminant analysis models. In case of image analysis of the images acquired through UAV various image processing techniques are explored and the features obtained from them for various precision agriculture applications are studied. Most of the machine learning models that are explored in case of weed classification and leaf disease detection does not incorporate UAV. Few of the studies in yield prediction and studies made on image analysis reveals that a different image analysis process is incorporated in case of UAV image analysis which differs from the traditional ones. It is believed that combining these would help future research to extend the applications of precision agriculture and also in getting better results.

\section{References}

[1] Piron A, Leemans V, Lebeau F, Destain MF. Improving in-row weed detection in multispectral stereoscopic images. Computers and electronics in agriculture. 2009 Nov 1;69(1):73-9.

[2] Bandi SR, Varadharajan A, Chinnasamy A. Performance evaluation of various statistical classifiers in detecting the diseased citrus leaves. International Journal of Engineering Science and Technology. 2013 Feb;5(2):298-307.

[3] Reshma R, Sathiyavathi V, Sindhu T, Selvakumar K, SaiRamesh L. IoT based Classification Techniques for Soil Content Analysis and Crop Yield Prediction. In2020 Fourth International Conference on ISMAC (IoT in Social, Mobile, Analytics and Cloud)(I-SMAC) 2020 Oct 7 (pp. 156-160). IEEE.

[4] Pandiyaraju V, Shunmuga Perumal P, Kannan A, Ramesh LS. Smart terrace gardening with intelligent roof control algorithm for water conservation. Pak. J. Agri. Sci. 2017 Jun 1;54(2):451-5.

[5] Reshma R, Sathiyavathi V, Sindhu T, Selvakumar K, SaiRamesh L. IoT based Classification Techniques for Soil Content Analysis and Crop Yield Prediction. In2020 Fourth International Conference on ISMAC (IoT in Social, Mobile, Analytics and Cloud)(I-SMAC) 2020 Oct 7 (pp. 156-160). IEEE.

[6] Pandiyaraju V, Shunmuga Perumal P, Sai Ramesh L, Ganapathy S, Kannan A. Dynamic Waypoint Navigation Assisted Agricultural Flying Vehicle for Field Data Collection. Asian Journal of Research in Social Sciences and Humanities. 2016;6(12):448-57.

[7] Xu D. Agricultural climate change based on remote sensing image and emergency material supply management of agriculture, rural areas and farmers. Arabian Journal of Geosciences. 2021 May;14(10):1-8.

[8] Agrawal H, Dhall R, Iyer KS, Chetlapalli V. An improved energy efficient system for IoT enabled precision agriculture. Journal of Ambient Intelligence and Humanized Computing. 2019 Jun 18:1-2. 